\title{
DIFFUSION NEAR AN ABSORBING POLYMER: MULTIFRACTAL DIMENSIONS
}

\author{
C. VON Ferber ${ }^{(1)}$, Yu.Holovatch ${ }^{(2)}$, L.SchäFeR, ${ }^{(1)}$ \\ (1) Fachbereich Physik der Universität Essen \\ D-45117 Essen, Germany \\ ${ }^{(2)}$ Institute for Condensed Matter Physics \\ Ukrainian National Academy of Sciences \\ 1 Svientsitskii St., 290011 Lviv, Ukraine
}

Received September 12, 1995

\begin{abstract}
We present some recent work on solutions of polymers of different composition say $A$ and $B$ with interactions $u_{A B}$ which may be linked together to form stars or networks and we discuss an application of this theory to a problem of diffusion limited aggregation.
\end{abstract}

\section{The Model}

Let us first take a look at the model we use to describe polymers. In a first discrete version we will describe a configuration of the polymer by a set of positions of segment endpoints:

$$
\text { Configuration }\left\{r_{1}, \ldots, r_{N}\right\} \in \mathbb{R}^{d \times N} .
$$

Its statistical weight (Boltzmann factor) with the Hamiltonian $\mathcal{H}$ divided by the product of Boltzmann constant $k_{\mathrm{B}}$ and temperature $T$ will be given by

$$
\exp \left[-\frac{1}{k_{\mathrm{B}} T} \mathcal{H}\right]=\exp \left\{-\frac{1}{4 \ell_{0}^{2}} \sum_{j=1}^{N}\left(r_{j}-r_{j-1}\right)^{2}-\beta \ell_{0}^{d} \sum_{i \neq j=1}^{N} \delta^{d}\left(r_{i}-r_{j}\right)\right\} .
$$

The first term describes the chain connectivity, the parameter $\ell_{0}$ governing the mean segment length. The second term describes the excluded volume interaction forbidding two segment end points to take the same position in space. The parameter $\beta$ gives the strength of this interaction. The third parameter in our model is the chain length or number of segments $N$.

The partition sum $\mathcal{Z}$ will be calculated as an integral over all configurations of the polymer divided by the system volume $\Omega$, thus dividing out identical configurations just translated in space $^{1}$.

$$
\mathcal{Z}(N)=\frac{1}{\Omega} \int \prod_{i=1}^{N} \mathrm{~d} r_{i} \exp \left[-\frac{1}{k_{\mathrm{B}} T} \mathcal{H}\left\{r_{i}\right\}\right] .
$$

\footnotetext{
${ }^{1}$ To give a meaning to the exponential of $\delta$-functions an appropriate short distance cutoff is introduced in the integrations.
}

(C) C. von Ferber, Yu.Holovatch, L.Schäfer, 1996

ISSN 0452-9910. Condensed Matter Physics 1996 No 7 (15-25) 
This will give us the 'number of configurations' of the polymer. It can be shown that for large $N$ the partition sum $\mathcal{Z}(N)$ is given by a power law

$$
\mathcal{Z}(N) \sim e^{\hat{\mu} N} N^{\gamma-1}, \quad N \rightarrow \infty .
$$

with a configuration number exponent $\gamma$ and a prefactor given by the number of configurations of a random walk with a 'chemical potential' $\hat{\mu}$ for the segments. The questions we want address here are

(1) Looking at linked polymers of possibly mixed composition, do we find such power laws again and what are the corresponding configuration number exponents?

(2) Diffusion near a fractal absorber.

- and of course: what has (2) to do with (1) ?

\section{Mapping the model onto a renormalizable field theory}

We will do our investigations by mapping the polymer model to a renormalizable field theory making use of well developed formalisms (see [1,2] for example). To this end we introduce a continuous version of our model as proposed by Edwards [3] generalizing it to describe a set of $l$ polymer chains of varying composition possibly tied together at their end points. The configuration of one polymer is now given by a path $r^{a}(s)$ in $d$ - dimensional space $\mathbb{R}^{d}$ parametrized by a surface variable $0 \leq s \leq S_{a}$. The relation of the 'Gaussian surface' $S_{a}$ of the chain $a$ to the number of segments $N_{a}$ in the discrete model is $S_{a}=N \ell_{0}^{2}$. We now allow for a symmetric matrix of excluded volume interactions $u_{a b}$ between chains $a, b=1, \ldots, l$. The Hamiltonian $\mathcal{H}$ is then given by

$$
\begin{aligned}
\frac{1}{k_{\mathrm{B}} T} \mathcal{H}= & \frac{1}{2} \sum_{a=1}^{l} \int_{0}^{S_{a}} \mathrm{~d} s\left(\frac{\mathrm{d} r^{a}(s)}{\mathrm{d} s}\right)^{2}+\frac{1}{2} \sum_{a, b=1}^{l} u_{a b} \int \mathrm{d}^{d} r \rho_{a}(r) \rho_{b}(r), \\
& \text { with densities } \quad \rho_{a}(r)=\int_{0}^{S_{a}} \mathrm{~d} s \delta^{d}\left(r-r^{a}(s)\right) .
\end{aligned}
$$

Now the partition sum has to be calculated using a functional integral. The partition sum $\mathcal{Z}_{l}$ for a polymer star, that is a set of $l$ polymers constrained to have a common end point is in these terms given by

$$
\mathcal{Z}_{l}\left\{S_{a}\right\}=\frac{1}{\Omega} \int \mathcal{D}\left[r^{a}(s)\right] \exp \left\{-\frac{1}{k_{\mathrm{B}} T} \mathcal{H}\left(r^{a}\right)\right\} \prod_{a=2}^{l} \delta^{d}\left(r^{a}(0)-r^{1}(0)\right) .
$$

The mapping to field theory is performed by a Laplace transform in the Gaussian surfaces $S_{a}$ to a conjugate chemical potential 'mass variable' $\mu_{a}$ :

$$
\tilde{\mathcal{Z}}_{l}\left\{\mu_{a}\right\}=\int \prod_{b} \mathbf{d} S_{b} e^{-\mu_{b} S_{b}} \mathcal{Z}_{l}\left\{S_{a}\right\} .
$$

In the corresponding Lagrangian field theory the transformed partition sum $\tilde{\mathcal{Z}}_{l}\left\{\mu_{a}\right\}$ can now be written as

$$
\tilde{\mathcal{Z}}_{l}\left\{\mu_{b}\right\}=\frac{1}{\Omega} \int \mathrm{d}^{d} r_{0} \prod_{a=1}^{l} \mathrm{~d}^{d} r_{a} \int \mathcal{D}\left[\phi_{a}(r)\right] \prod_{a=1}^{l} \phi_{a}\left(r_{0}\right) \phi_{a}\left(r_{a}\right) \exp \left[-\mathcal{L}\left\{\phi_{b}, \mu_{b}\right\}\right],
$$


in the limit of $m=0$ component fields $\phi_{a}$. The Landau Ginzburg Wilson Lagrangian $\mathcal{L}$ of $l$ interacting fields $\phi_{b}$ each with $m$ components reads

$$
\begin{gathered}
\mathcal{L}\left\{\phi_{b}, \mu_{b}\right\}=\frac{1}{2} \sum_{a=1}^{l} \int \mathrm{d}^{d} r\left(\mu_{a} \phi_{a}^{2}+\frac{1}{2}\left(\nabla \phi_{a}(r)\right)^{2}\right) \\
+\frac{1}{8} \sum_{a, a^{\prime}=1}^{l} u_{a, a^{\prime}} \int \mathrm{d}^{d} r \phi_{a}^{2}(r) \phi_{a^{\prime}}^{2}(r) .
\end{gathered}
$$

In components $\phi_{a, \alpha}$ with $\alpha=1, \ldots, m$ the scalar products are written as

$$
\phi_{a}^{2}=\sum_{\alpha=1}^{m} \phi_{a, \alpha}^{2} .
$$

The formal limit $m=0$ in (2.4) eliminates the bubble graph contributions which have combinatorial factors of powers of $m$ thus producing the 'polymer limit' of $\phi^{4}$ field theory. As is well known $[5,6]$ ultraviolet divergencies occur evaluating this theory naively. These can be removed by ...

\section{Renormalization}

\subsection{A single polymer $(l=1)$}

In the case of a single polymer a renormalized field $\phi_{1, R}$, a renormalized mass parameter $t_{1}$ and a renormalized coupling $g$ may be constructed at a momentum scale $\kappa$ of renormalized theory. Appropriate $Z$ - factors are introduced as power series in the renormalized coupling $g$ absorbing the ultraviolet divergencies of bare theory:

$$
\begin{gathered}
\phi_{1}(r)=Z^{1 / 2}(g) \phi_{1, R}(r), \\
\mu_{1}-\mu_{\text {crit }}=\kappa^{2} \frac{Z_{2}(g)}{Z(g)} t_{1}, \\
u_{11}=\kappa^{4-d} \frac{Z_{4}(g)}{Z^{2}(g)} g .
\end{gathered}
$$

Under rescaling of $\kappa$ a nontrivial fixed point of renormalized theory at $g=$ $g^{*} \neq 0$ is found. The multiplicative renormalizations in (3.1-3.3) result in power law scaling at the fixed point. In the Laplace transformed theory a power law for the number of configurations can be derived in the limit of long chains $S_{1} \rightarrow \infty$ :

$$
\mathcal{Z}\left(S_{1}\right) \sim \exp \left[\mu_{\text {crit }} S_{1}\right] S_{1}^{\gamma-1}
$$

The configuration number exponent $\gamma$ is shown to be the critical susceptibility exponent of the $m$ vector model in the limit $m=0$.

\subsection{An $l$ arm star polymer}

Considering the partition of a star polymer with $l$ arms sum we see that the field theoretic functional integral contains a composite operator $\prod_{a=1}^{l} \phi_{a}\left(r_{0}\right)$. It can be shown that this operator is renormalized multiplicatively $[4,7]$

$$
\prod_{a=1}^{l} \phi_{a}\left(r_{0}\right)=Z^{l / 2}(g) Z_{* l}^{-1}(g)\left[\prod_{a=1}^{l} \phi_{a}\left(r_{0}\right)\right]_{R}
$$




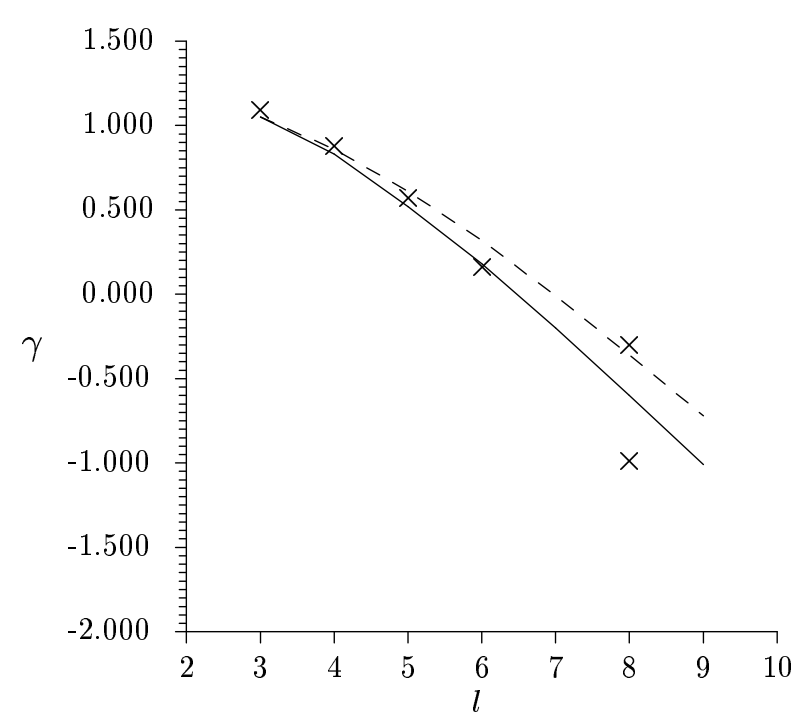

Figure 1. Configuration number exponent $\gamma_{l}=\nu \eta_{l}+1$ as function of $l$ calculated for the symmetric case by resummed $\epsilon^{3}$-expansion [4] (solid curve), by resummation of three-loop renormalization group functions directly in three dimensions $[15,16]$ (dashed curve) in comparison with Monte-Carlo data [13,14] (crosses).

without affecting the renormalizations (3.1-3.3) described above. In addition in the $m=0$ limit no coupling occurs between the mass parameters $t_{a}$ of the different fields. Thus there is no change to the fixed point and we can again find a power law behaviour for the partition sum in the limit of long chains. If all chains are of equal size $R=S_{a}^{\nu}$ for $a=1, \ldots, l$ we have

$$
\mathcal{Z}_{l}(S) \sim R^{\eta_{l}} \exp \left[\mu_{\text {crit }} \sum_{a} S_{a}\right]
$$

with a family of exponents $\eta_{l}, l=1,2,3,4, \ldots$. These exponents form a linear basis for the configuration number exponents of networks [8].

\subsection{Two components of polymers}

Let us now consider the case of a polymer star consisting of $l_{1}$ arms of polymers of a first kind and $l_{2}$ arms of polymers of a second kind. The interaction between polymers of 1 st kind shall be $u_{11}^{0}$ for the 2 nd kind polymers $u_{22}^{0}$, the interaction between polymers of 1st kind and those of 2nd kind shall be $u_{12}^{0}$. The matrix of interactions then reads

$$
u_{a b}=\left\{\begin{array}{llrl}
u_{11}^{0} & \text { for } & a, b \leq l_{1} \\
u_{12}^{0} & \text { for } \quad a \leq l_{1}<b \text { or } b \leq l_{1}<a \\
u_{22}^{0} & \text { for } & & l_{1}<a, b .
\end{array}\right.
$$

In the renormalized theory for such systems one finds additional nontrivial fixed points for the corresponding renormalized couplings $g_{11}, g_{12}, g_{22}$. In 
the three dimensional space of these couplings the 8 fixed points are found at the corners of a cube slightly deformed in $g_{12}$ direction [9]. The interesting fixed points are those with non vanishing interaction $g_{12}$ between the two kinds of polymers. These fixed points are in coordinates $P=\left(g_{11}^{*}, g_{22}^{*}, g_{12}^{*}\right)$ :
S: 'symmetric'
$S=\left(g^{*}, g^{*}, g^{*}\right)$
$\mathrm{U}:$ 'asymmetric'
$U=\left(g^{*}, 0, g_{U}^{*}\right)$
$G=\left(0,0, g_{G}^{*}\right)$
and $\quad U^{\prime}=\left(0, g^{*}, g_{U}^{*}\right)$
G: 'gaussian'

For polymer stars described above one finds additional families of configuration number exponents for these fixed points:

$\eta_{l_{1} l_{2}}^{G}$ : the 'Gaussian' exponent describes a star of $l_{1}$ chains of 1 st kind and $l_{2}$ chains of 2 nd kind which are all non selfavoiding, only chains of 1 st kind avoid those of 2nd kind and vice versa.

$\eta_{l_{1} l_{2}}^{U}$ : the 'asymmetric' exponent describes a star of $l_{1}$ self and mutually avoiding polymer chains of 1 st kind and $l_{2}$ chains which only avoid those of 1st kind.

$\eta_{l_{1} l_{2}}^{S}$ : the 'symmetric' exponent describes a star of $l_{1}+l_{2}$ self and mutually avoiding chains. This is the situation we have already discussed before: $\eta_{l_{1} l_{2}}^{S}=\eta_{l_{1}+l_{2}}$ (see equation (3.6)).

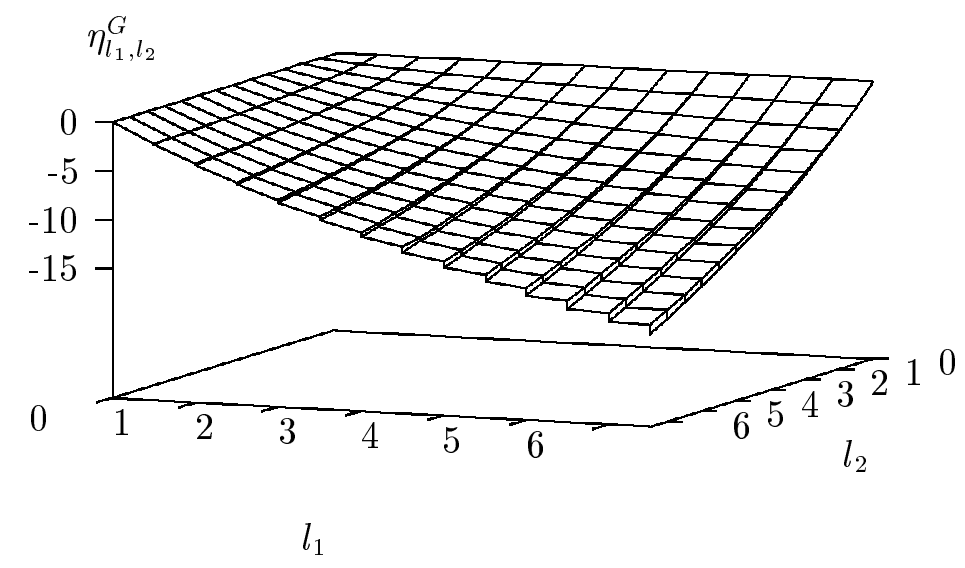

Figure 2. Exponent $\eta_{l_{1} l_{2}}^{G}$ in the 'Gaussian' fixed point at $d=2$ obtained in $\epsilon$-expansion and in fixed $d$ scheme.

\section{The fractal absorber model}

We will now turn to a model proposed by Cates and Witten [10] and which is in close connection to our previous considerations as we will see below. We want to describe the flow of diffusing particles near an absorbing object, called 'absorber', which may be a fractal. More generally we are looking at scalar solutions of the Laplace equation

$$
\nabla^{2} u(r)=0
$$


with boundary conditions for a given absorbing object

$$
\begin{array}{lc}
u(r)=0 & \text { on the surface of the absorber } \\
u(r)=u_{\infty} & \text { for } \quad|r| \rightarrow \infty .
\end{array}
$$

We will here consider the special case of the absorbing object being

a) a random walk (RW)

b) a polymer or self avoiding walk (SAW) .

A more physical interpretation of this model may be found by letting $u(r)$ describe the probability density of diffusing particles $A$ which will be transformed to particles $A^{\prime}$ by a process $A \rightarrow A^{\prime}$ catalysed by the fractal object.

\subsection{The solution}

Let the absorber be described by the path $r^{a}(s), 0 \leq s \leq S_{a}$. In terms of the path integral solution of the Laplace equation we find that $u(\rho)$ at a point $\rho$ near the absorber is proportional to the number of random walks

$$
\mathcal{Z}_{1}^{0}\left(\rho, r^{a}\right)
$$

which end at point $\rho$ and avoid the absorber. Also higher moments of $u(\rho)$ at some distance $x$ from a fixed point on the absorber can be described in the same way.

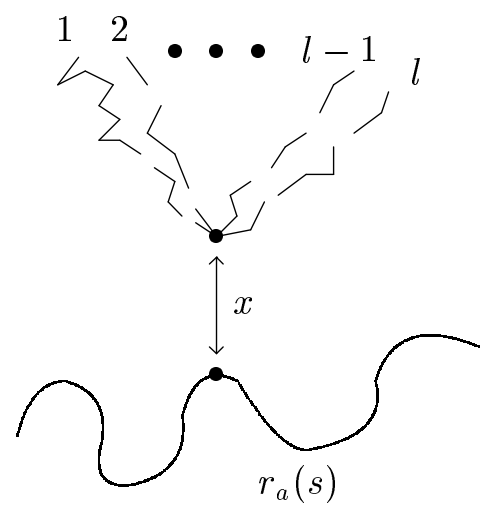

Figure 3. Star of $l$ random walks at distance $x$ from polymer absorber $r_{a}(s)$.

Let the $l$-th moment of $u(\rho)$ at distance $x$ from some point on the absorber be denoted by

$$
\left\langle u^{l}(x)\right\rangle
$$

where we take the mean value over all configurations $\left\{r^{a}(s)\right\}$ of the absorber and all points $r^{a}\left(s_{0}\right)$ on the absorber. Then $\left\langle u^{l}(x)\right\rangle$ is proportional to the configurations of a random walk star at distance $x$ from a polymer. For a solution of this problem we will consider the limiting procedure $x \rightarrow 0$ in which case we can interpret $x$ as the minimal distance of the star vertex to the polymer. The situation described above may be brought back to case of single star polymers by results of a short distance expansion. 
We find:

The $l$ th moments of the 'density' distribution $u(r)$ near the absorber is described by polymer star exponents. The moments scale with the distance $x$ from some point on the absorber and the absorber size $R$ as

$$
\left\langle u^{l}(x)\right\rangle \sim(x / R)^{\lambda_{l}} \quad \text { for } \quad(x / R) \rightarrow 0
$$

If the absorber is

a) a RW

$$
\lambda_{l}=-\eta_{2 l}^{G}=\epsilon l\left(1-\frac{l-1}{4} \epsilon+\frac{l-1}{8}(l-1+3 \zeta(3)) \epsilon^{2}+\ldots\right)
$$

b) a SAW

$$
\begin{aligned}
\lambda_{l}= & -\eta_{2 l}^{U}+\eta_{2}=\frac{3}{4} \epsilon l\left(1-\frac{3 l-7 / 6}{16} \epsilon+\right. \\
& \left.\left(9 l^{2}-\frac{7}{2} l-\frac{149}{12}+45 l \zeta(3)-23 \zeta(3)\right) \frac{\epsilon^{2}}{128}+\ldots\right) .
\end{aligned}
$$

Already in second order of $\epsilon$ we see that the exponents have a nontrivial dependence on the order $l$ of the moment considered. We have here a real multifractal phenomenon.

\subsection{Fractal measure exponents and spectral function}

Different formalisms were introduced for description of multifractal phenomena. Thus in order to describe properties of 'harmonic measure' $\mu(x)$ which in our case is associated with a flux $\phi(x)$ of a Laplasian field $u(r)$ by the relation

$$
\mu(x)=\phi(x) / \sum \phi(x),
$$

(here and below $\sum$ means sum over all particles (being of size $a$ ) of the absorber) one can define the set of exponents $D(l)$ by [11]:

$$
\sum \mu(x)^{l}=(R / a)^{(1-l) D(l)} .
$$

In the case $l=0 D(0)$ being fractal dimension of the absorber: in our case for $R W$

$$
D^{R W}(0)=2,
$$

for SAW in three dimensions

$$
D^{S A W}(0)=1 / \nu^{S A W}(d=3) \simeq(0.588)^{-1} \simeq 1.7 .
$$

On the base of definitions (4.6), (4.11) one can obtain [10] the following relation between exponents $\lambda(l)$ and $D(l)$ :

$$
l \lambda(1)-\lambda(l)=(1-l)[D(l)-D(0)] .
$$




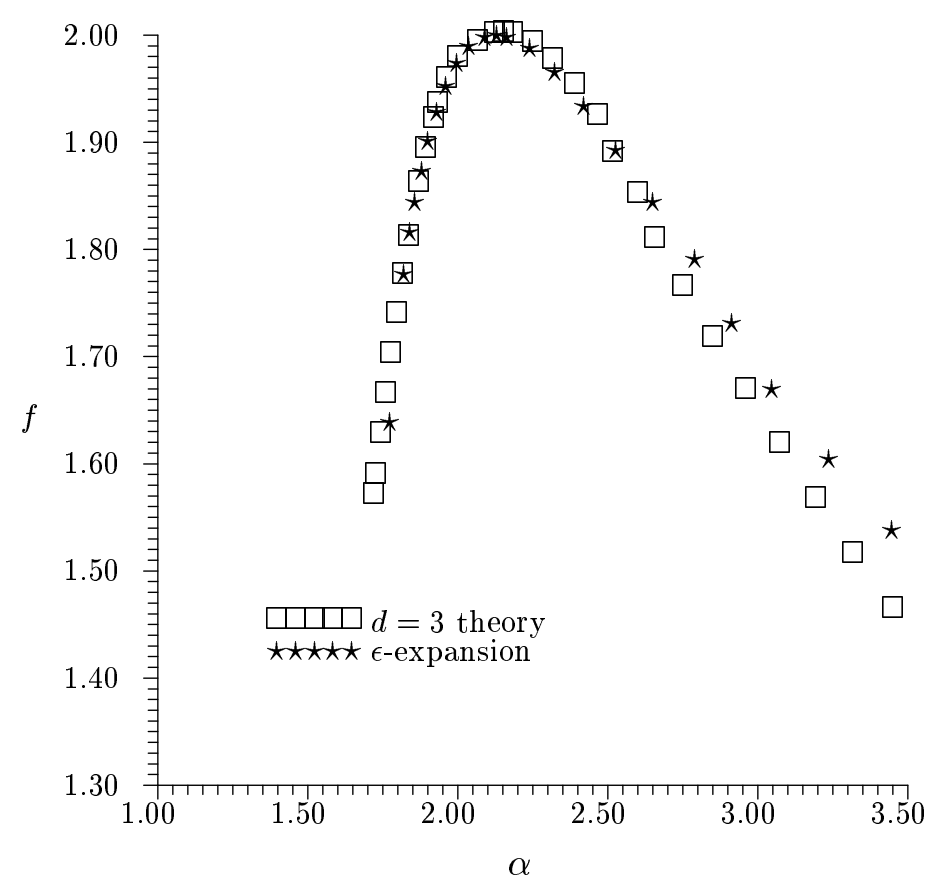

Figure 4. Spectral function $f(\alpha)$ for absorbtion on $R W$.

In many cases description is performed in terms of spectral function $f(\alpha)$ which is essentially a Legendre transform of $D(l)$ given by:

$$
\begin{aligned}
\alpha(l) & =\frac{d}{d l}[(l-1) D(l)], \\
f(\alpha) & =l \alpha(l)+(1-l) D(l)
\end{aligned}
$$

Function $f(\alpha)$ differs for different fractal measures but it possess certain 'universal' properties [12]: calculation of this function and analysis of its properties is also the subject of this study.

An application of these results can be found for the reaction rate of a process

$$
l \cdot A \rightarrow B
$$

where $l$ particles of type $A$ react to the product $B$ catalyzed by the fractal object. It can be shown [10] that the reaction rate $Q(l)$ for this reaction scales with the size $R$ of the object as

$$
Q(l) \sim R^{D-\lambda_{l}}
$$

where $D=\frac{1}{\nu}$ is the fractal (Hausdorff) dimension of the catalyzer. Taking into account that starting from a certain value of $l \lambda_{l}$ becomes negative we see that the reaction rate is enhanced considerably for the fractal random walk absorber as compared to an absorber in form of a sphere which has the same dimension $D=2$. 


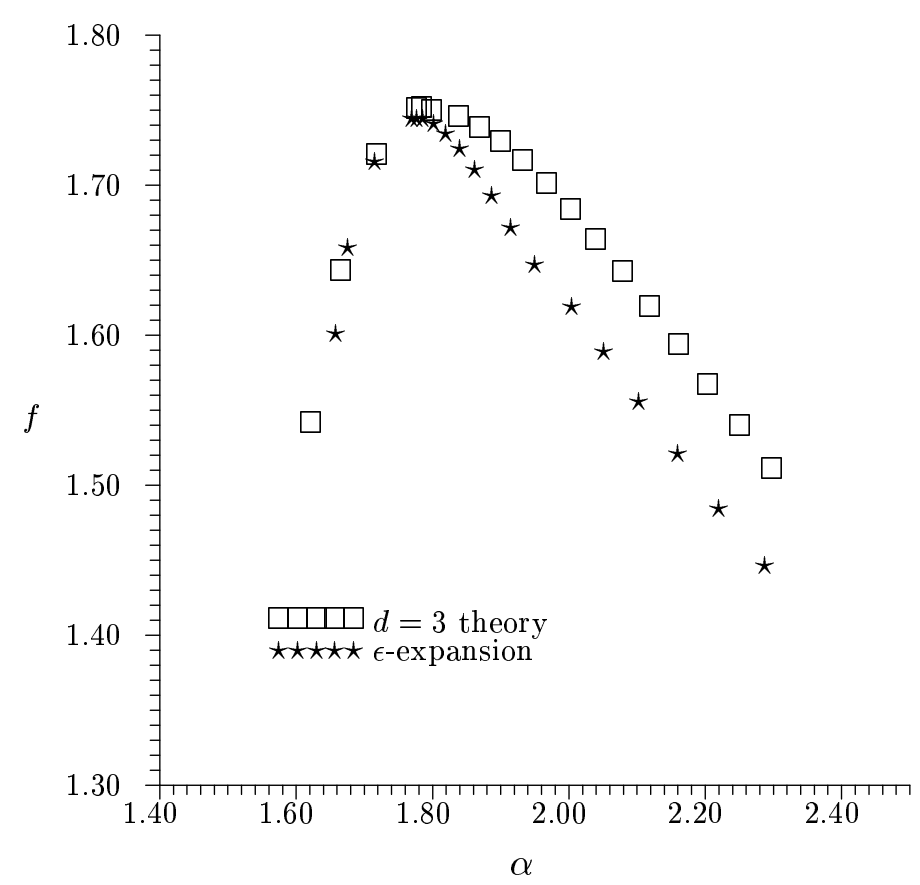

Figure 5. Spectral function $f(\alpha)$ for absorbtion on $S A W$.

\section{Results}

For the symmetric case the resummation of the $\epsilon$ expansion is shown in figure 1 together with Monte - Carlo simulation results [13,14]. We have plotted the configuration number exponent $\gamma_{l}=\nu \eta_{l}+1$ in equation (3.6) for $l=3, \ldots, 9$ using the resummation procedure based on the conformal mapping technique. In a present study we are extending these calculations to the before described asymmetric and Gaussian cases. Also we are investigating the influence of the choice of the renormalization procedure. The first procedure was dimensional regularization and renormalization in $\epsilon$ expansion. In a second approach we use a massive renormalization scheme with renormalization at fixed dimension in a scheme $[15,16]$ which generalizes the idea of [17]. Resummed results of this second approach are also shown in figure 1 in comparison to the other data. For higher numbers $l$ of arms of the polymer star one can not expect this perturbative approach to be too good even renormalized. The reason is that the combinatorial factors lead to an expansion in $l \cdot \epsilon$ for the $\epsilon$ expansion or $l \cdot g$ when directly expanding in a renormalized coupling $g$. Therefore the case of high order moments or high order of arms is to be studied in a separate approach using a self consistent field formalism [10].

Figure 2 shows a 'flying carpet' describing the dependence of the exponent $\eta_{l_{1} l_{2}}^{G}$ in $d=2$ in the 'Gaussian' fixed point obtained in $\epsilon$-expansion and in the fixed dimension scheme. Difference in the results of both approaches appear as 'steps' in the carpet.

Figures 4, 5 show the resummed function $f(\alpha)$ in the case of the absorber being a $R W$ (figure 4 ) and a $S A W$ (figure 5) (stars showing results of $\epsilon$ - 
expansion, squares correspond to the massive renormalization group scheme in three dimensions). The maximum value of $f(\alpha)$ corresponds to the fractal dimension of the absorber given by (4.12) (figure 4) and (4.13) (figure 5). The resummation procedure applied to the pertubation theory series enables us to obtain reliable data not only in the region of $\alpha$ corresponding to $l$ close to zero, as it was done in [10] but to proceed to higher values of $l$ as well 2. Furthermore we have shown how to derive the multifractal spectrum from the spectrum of appropriately chosen operators in a Lagrangean field theory. This aspect will be subject of a forthcoming publication.

\section{Acknowledgements}

This work has been supported in part by the Deutsche Forschungsgemeinschaft, Sonderforschungsbereich "Unordnung und große Fluktuationen" and by the Ukrainian State Committee for Science and Technology, project No $2.3 / 665$.

\section{References}

[1] De Gennes P.G. Scaling concepts in polymer physics. New York, Ithaca, Cornell University Press, 1979.

[2] des Cloizeaux J., Jannik G. Les polymères en solution: Leur modélization et leur structure. Les Ulis: Les éditions de physique, 1987.

[3] Edwards S.F. // Proc. Phys. Soc., 1965, vol. 85, p. 613; Proc. Phys. Soc., 1966, vol. 88, p. 265.

[4] Schäfer L., von Ferber C., Lehr U., Duplantier B. Renormalization of polymer networks and stars. // Nucl. Phys. B, 1992, vol. 374, No 3, p. 473-495.

[5] Bogoliubov N. N., Shirkov D. V. Introduction to the theory of quantized fields. New York, Wiley, 1959.

[6] Brezin E., Le Guillou J.C., Zinn-Justin J. Field theoretical approach to critical phenomena. In: "Phase transitions and critical phenomena", ed. by C.Domb and M.S.Green. New York, Academic Press, 1976, vol. 6, p. 125-247.

[7] Wallace D.J., Zia R.K.P. Harmonic perturbations of generalized Heisenberg spin systems. // J. Phys., 1975, vol. C8, p. 839-843.

[8] Duplantier B. Statistical mechanics of polymer networks of any topology. // J. Stat. Phys., 1989, vol. 54, No 3/4, p. 581-680.

[9] Schäfer L., Lehr U., Kappeler C. Higher order calculations of the renormalization group flow for multicomponent polymer solutions. // J. Phys. I (Paris), 1991, vol. 1, No 1, p. 211-233.

[10] Cates M.E., Witten T.A. Diffusion near absorbing fractals: Harmonic measure exponents for polymers. // Phys. Rev. A, 1987, vol. 35, p. 1809-1824.

[11] Hentschel H.G.E., Procaccia I. The infinite number of generalized dimensions of fractals and strange attractors. // Physica D, 1983, vol. 8, p. 435-444.

[12] Halsey T.C., Jensen M.H., Kadanoff L.P., Procaccia I., Shraiman B.I. Fractal measures and their singularities: The characterization of strange sets. // Phys. Rev. A, 1986, vol. 33, No 2, p. 1141-1151.

[13] Batoulis J., Kremer K. Thermodynamic properties of star polymers: good solvents. // Macromolecules, 1989, vol. 22, p. 4277-4285.

[14] Barret A.J., Tremain T.L. Lattice walk models of star polymers with many arms. // Macromolecules, 1987, vol. 20, p. 1687-1692.

[15] von Ferber C., Holovatch Yu. Star exponents in polymer theory: renormalization group results in three dimensions. // Cond. Matt. Phys. (Lviv), 1995, No 5, p. 8-22.

\footnotetext{
${ }^{2}$ Note that resummation is performed both for series $\alpha(l)$ and $f(l)$.
} 
[16] von Ferber C., Holovatch Yu. Polymer stars in three dimensions. Three loop results. // Theor. Mat. Phys. (Moscow), 1995 (to be published).

[17] Parisi G. Field-theoretic approach to second-order phase transitions in twoand three-dimensional systems. // J. Stat. Phys., 1980, vol. 23, No 1, p. 49-82.

\title{
ДИФУЗІЯ БІЛЯ АБСОРБУЮЧОГО ПОЛІМЕРА: МУЛЬТИФРАКТАЛЬНІ ВИМІРНОСТІ
}

\author{
К. фон Фербер, Ю.Головач, Л.Шефер
}

Досліджуються розчини полімерів різних сортів (напр. $A$ і $B)$ із взаємодіями $U_{A B}$, котрі можуть бути з'єднані і утворювати зірки або сітки. Обсуджується застосування теорії до проблеми DLA (обмеженої дифузією агрегації). 
